\title{
ON THE NONEXISTENCE OF A LAW OF THE ITERATED LOGARITHM FOR WEIGHTED SUMS OF IDENTICALLY DISTRIBUTED RANDOM VARIABLES ${ }^{1}$
}

\author{
André Adler \\ Illinois Institute of Technology \\ Chicago, IL 60616
}

\begin{abstract}
For weighted sums of independent and identically distributed random variables, conditions are placed under which a generalized law of the iterated logarithm cannot hold, thereby extending the usual nonweighted situation.
\end{abstract}

AMS Subject Classification: 60F15.

Key Words: law of the iterated logarithm, strong law of large numbers.

\section{INTRODUCTION.}

Heyde [1] established the fact that partial sums of independent and identically distributed (i.i.d.) random variables $\left\{X, X_{n}, n \geq 1\right\}$ whose common distribution is of the form $P\{|X|>$ $x\}=L(x) x^{-\alpha}(0 \leq \alpha<2, \alpha \neq 1)$, where $L(x)$ is slowly varying at infinity and where $E X=0$ if $E|X|<\infty$, cannot be normalized in the sense that there exist constants $0<b_{n} \uparrow$ with $\sum_{k=1}^{n} X_{k} / b_{n} \rightarrow 1$ a.s. The purpose of this paper is to present similar results in the weighted case.

Herein, we define $S_{n}=\sum_{k=1}^{n} a_{k} X_{k}$ where $\left\{a_{n}, n \geq 1\right\}$ are constants and the random variables $\left\{X, X_{n}, n \geq 1\right\}$ are identically distributed with common distribution

$$
P\{|X|>x\}= \begin{cases}L(x) x^{-\alpha} & x \geq 1, \\ 1 & x<1\end{cases}
$$

where $L(c x) / L(x) \rightarrow 1$ as $x \rightarrow \infty$ for all $c>0$, and $\alpha \geq 0$.

A remark about notation is needed. Throughout, the symbol $C$ will denote a generic finite nonzero constant which is not necessarily the same in each appearance. Also, we let $c_{n}=b_{n} /\left|a_{n}\right|, n \geq 1$, where $\left\{b_{n}, n \geq 1\right\}$ is our norming sequence.

It should be noted that the techniques involved with the main results (Theorems 2 and 3) follow a similar pattern to those that can be found in Heyde [1]. As usual, via the BorelCantelli lemma, one need only consider a truncated version of the random variables $\left\{X_{n}\right.$, $n \geq 1\}$. Instead of truncating $X_{n}$ at $b_{n}$ the trick, in the weighted case, is to cut off $X_{n}$ at $c_{n}$. Then by classical arguments the remaining terms are shown to be almost surely negligible. Also of particular interest is the discussion (Section 3) of the $\alpha=1$ situation.

${ }^{1}$ Received: January 1989; Revised: December 1989 


\section{RESULTS.}

Our first theorem examines what happens when $P\left\{\left|X_{n}\right|>c_{n}\right.$ i.o. $\left.(n)\right\}=1$.

Theorem 1. Let $\left\{X, X_{n}, n \geq 1\right\}$ be i.i.d. random variables. If $\left\{a_{n}, n \geq 1\right\}$ and $\left\{b_{n}\right.$, $n \geq 1\}$ are constants satisfying $b_{n}=O\left(b_{n+1}\right), b_{n} \rightarrow \infty$, and $\sum_{n=1}^{\infty} P\left\{|X|>c_{n}\right\}=\infty$, then $\lim \sup _{n \rightarrow \infty}\left|S_{n}\right| / b_{n}=\infty$ a.s.

Proof. If $c_{n} \rightarrow \infty$, then for all large $M$

$$
\begin{aligned}
\sum_{n=1}^{\infty} P\left\{\left|a_{n} X_{n}\right|>M b_{n}\right\} & =\sum_{n=1}^{\infty} L\left(M c_{n}\right)\left(M c_{n}\right)^{-\alpha} \\
& \geq C \sum_{n=1}^{\infty} L\left(c_{n}\right) c_{n}^{-\alpha} \\
& \geq C \sum_{n=n_{0}}^{\infty} P\left\{\left|X_{n}\right|>c_{n}\right\} \text { (for a suitably chosen } n_{0} \text { ) } \\
& =\infty .
\end{aligned}
$$

Otherwise, if $\lim \inf _{n \rightarrow \infty} c_{n}<\infty$, then there exists a subsequence $\left\{n_{k}, k \geq 1\right\}$ and a finite constant $B$ such that $c_{n_{k}} \leq B$. Hence for all $0<M<\infty$

$$
\begin{aligned}
\sum_{n=1}^{\infty} P\left\{|X|>M c_{n}\right\} & \geq \sum_{k=1}^{\infty} P\left\{\left|X_{n}\right|>M c_{n_{k}}\right\} \\
& \geq \sum_{k=1}^{\infty} P\{|X|>M B\} \\
& =\infty .
\end{aligned}
$$

So in either case we conclude, via the Borel-Cantelli lemma, that

$$
\lim \sup _{n \rightarrow \infty}\left|\frac{a_{n} X_{n}}{b_{n}}\right|=\infty \text { a.s. }
$$

Since

$$
\left|\frac{a_{n} X_{n}}{b_{n}}\right| \leq\left|\frac{S_{n}}{b_{n}}\right|+\left|\frac{b_{n-1}}{b_{n}}\right| \cdot\left|\frac{S_{n-1}}{b_{n-1}}\right|
$$

the conclusion follows.

Note that in the next result independence is not necessary.

Theorem 2. Let $\left\{X, X_{n}, n \geq 1\right\}$ be identically distributed random variables. Let $\left\{a_{n}\right.$, $n \geq 1\}$ and $\left\{b_{n}, n \geq 1\right\}$ be constants satisfying $0<b_{n} \uparrow \infty$ and $\sum_{n=1}^{\infty} P\left\{|X|>c_{n}\right\}<\infty$. If $0 \leq \alpha<1$, then $S_{n} / b_{n} \rightarrow 0$ a.s.

Proof. Notice, via the Borel-Cantelli lemma, that

$$
\sum_{k=1}^{n} a_{k} X_{k} I\left(\left|X_{k}\right|>c_{k}\right)=o\left(b_{n}\right) \text { a.s. }
$$

Hence it remains to show that

$$
\sum_{k=1}^{n} a_{k} X_{k} I\left(\left|X_{k}\right| \leq c_{k}\right)=o\left(b_{n}\right) \text { a.s. }
$$


Since, for all large $k$

$$
\begin{aligned}
E|X| I\left(|X| \leq c_{k}\right) & \leq \int_{0}^{c_{k}} P\{|X|>t\} d t \\
& =\int_{0}^{1} d t+\int_{1}^{c_{k}} L(t) t^{-\alpha} d t \\
& \leq C L\left(c_{k}\right) c_{k}^{-\alpha+1}
\end{aligned}
$$

(by Theorem $1 \mathrm{~b}$ of Feller [2, p. 281]), it follows that

$$
\begin{aligned}
\sum_{k=1}^{\infty} c_{k}^{-1} E|X| I\left(|X| \leq c_{k}\right) & \leq C \sum_{k=1}^{\infty} L\left(c_{k}\right) c_{k}^{-\alpha} \\
& \leq C \sum_{k=1}^{\infty} P\left\{|X|>c_{k}\right\} \\
& <\infty,
\end{aligned}
$$

whence

$$
\sum_{k=1}^{\infty} c_{k}^{-1}\left|X_{k}\right| I\left(\left|X_{k}\right| \leq c_{k}\right)<\infty \text { a.s. }
$$

This, via Kronecker's lemma, implies (1).

Next, we examine the mean zero situation.

Theorem 9. Let $\left\{X, X_{n}, n \geq 1\right\}$ be i.i.d. mean zero random variables. Let $\left\{a_{n}, n \geq 1\right\}$ and $\left\{b_{n}, n \geq 1\right\}$ be constants satisfying $0<b_{n} \uparrow \infty$ and $\sum_{n=1}^{\infty} P\left\{|X|>c_{n}\right\}<\infty$. If $1<\alpha<2$, then $S_{n} / b_{n} \rightarrow 0$ a.s.

Proof. Again, note that

$$
\sum_{k=1}^{n} a_{k} X_{k} I\left(\left|X_{k}\right|>c_{k}\right)=o\left(b_{n}\right) \text { a.s. }
$$

Since

$$
\begin{aligned}
\sum_{k=1}^{n} a_{k} X_{k}= & \sum_{k=1}^{n} a_{k}\left[X_{k} I\left(\left|X_{k}\right| \leq c_{k}\right)-E X I\left(|X| \leq c_{k}\right)\right] \\
& +\sum_{k=1}^{n} a_{k} E X I\left(|X| \leq c_{k}\right)+\sum_{k=1}^{n} a_{k} X_{k} I\left(\left|X_{k}\right|>c_{k}\right)
\end{aligned}
$$

we need only show that the first two terms are $o\left(b_{n}\right)$. In view of the Khintchine-Kolmogorov convergence theorem and Kronecker's lemma, all that one needs to show, in order to prove that the first term is $o\left(b_{n}\right)$ a.s., is that

$$
\sum_{k=1}^{\infty} c_{k}^{-2} E X^{2} I\left(|X| \leq c_{k}\right)<\infty
$$

By integration by parts and Theorem $1 \mathrm{~b}$ of Feller [2, p. 281] we observe that

$$
\sum_{k=1}^{\infty} c_{k}^{-2} E X^{2} I\left(|X| \leq c_{k}\right) \leq 2 \sum_{k=1}^{\infty} c_{k}^{-2} \int_{0}^{c_{k}} t P\{|X|>t\} d t
$$




$$
\begin{aligned}
& \leq C \sum_{k=1}^{\infty} L\left(c_{k}\right) c_{k}^{-\alpha} \\
& \leq C \sum_{k=1}^{\infty} P\left\{|X|>c_{k}\right\} \\
& <\infty .
\end{aligned}
$$

Hence (2) holds. Finally, we need to show that

$$
\sum_{k=1}^{n} a_{k} E X I\left(|X| \leq c_{k}\right)=o\left(b_{n}\right) .
$$

Due to the fact that $\left|E X I\left(|X| \leq c_{k}\right)\right| \leq E|X| I\left(|X|>c_{k}\right)$ it is sufficient to show that

$$
\sum_{k=1}^{n}\left|a_{k}\right| E|X| I\left(|X|>c_{k}\right)=o\left(b_{n}\right) .
$$

However, since

$$
\begin{aligned}
\sum_{k=1}^{\infty} c_{k}^{-1} E|X| I\left(|X|>c_{k}\right) & =\sum_{k=1}^{\infty} P\left\{|X|>c_{k}\right\}+\sum_{k=1}^{\infty} c_{k}^{-1} \int_{c_{k}}^{\infty} P\{|X|>t\} d t \\
& \leq O(1)+C \sum_{k=1}^{\infty} c_{k}^{-1} \int_{c_{k}}^{\infty} L(t) t^{-\alpha} d t \\
& \leq O(1)+C \sum_{k=1}^{\infty} L\left(c_{k}\right) c_{k}^{-\alpha} \text { (see Feller, [2, p.281]) } \\
& \leq O(1)+C \sum_{k=1}^{\infty} P\left\{|X|>c_{k}\right\} \\
& =O(1),
\end{aligned}
$$

it is clear that (3) obtains.

\section{DISCUSSION.}

In this section we combine the previous theorems. The conclusion is that for all $\alpha \in$ $[0,1) \cup(1,2)$ a law of the iterated logarithm cannot hold.

Theorem 4. Let $\left\{X, X_{n}, n \geq 1\right\}$ be i.i.d. random variables with

$$
P\{|X|>x\}= \begin{cases}L(x) x^{-\alpha} & x \geq 1 \\ 1 & x<1\end{cases}
$$

with $E X=0$ if $\alpha>1$. If $\left\{a_{n}, n \geq 1\right\}$ and $\left\{b_{n}, n \geq 1\right\}$ are constants with $0<b_{n} \uparrow \infty$, then for all $\alpha \in[0,1) \cup(1,2)$

$$
\lim \sup _{n \rightarrow \infty}\left|\frac{\sum_{k=1}^{n} a_{k} X_{k}}{b_{n}}\right|=0 \text { or } \infty \text { a.s. }
$$


depending on whether $\sum_{n=1}^{\infty} P\left\{|X|>c_{n}\right\}$ converges or diverges.

Proof. In view of Theorems 1, 2, and 3 the conclusion is immediate.

Now, clearly if a law of the iterated logarithm does not exist, then a strong law of large numbers (with limit one) is also not feasible.

Corollary. If the hypotheses of Theorem 4 hold, then

$$
P\left\{\lim _{n \rightarrow \infty} \sum_{k=1}^{n} a_{k} X_{k} / b_{n}=1\right\}=0 .
$$

It is well known that if $\alpha>2$, then a classical law of the iterated logarithm can be obtained provided suitable conditions are imposed on the constants $\left\{a_{n}, n \geq 1\right\}$. An interesting question is what happens when $\alpha=1$. If we allow $\alpha=1$, then not only can a law of the iterated logarithm obtain, but a strong law of large numbers can also occur where the limit is one. The following example is of the flavor of those that can be found in Adler [3].

Example. If $\left\{X_{n}, n \geq 1\right\}$ are i.i.d. random variables with common density $f(x)=$ $x^{-2} I_{(1, \infty)}(x),-\infty<x<\infty$, then

$$
\frac{\sum_{k=1}^{n} \frac{2}{k} X_{k}}{(\log n)^{2}} \rightarrow 1 \text { a.s. }
$$

Proof. Since

$$
\sum_{n=1}^{\infty} P\left\{|X|>\frac{n(\log n)^{2}}{2}\right\}=2+\sum_{n=3}^{\infty} \frac{2}{n(\log n)^{2}}<\infty
$$

and

$$
\left[\frac{n(\log n)^{2}}{2}\right]^{2} \sum_{j=n}^{\infty}\left[\frac{2}{j(\log j)^{2}}\right]^{2}=O(n)
$$

we have, by Theorem 1 of Adler and Rosalsky [4],

$$
\frac{\sum_{k=1}^{n} \frac{2}{k}\left(X_{k}-\mu_{k}\right)}{(\log n)^{2}} \rightarrow 0 \text { a.s. }
$$

where

$$
\begin{aligned}
\mu_{n} & =E X I\left(|X| \leq \frac{n(\log n)^{2}}{2}\right) \\
& =\int_{1}^{n(\log n)^{2} / 2} x^{-1} d x \\
& \sim \log n .
\end{aligned}
$$

Noting that

$$
\frac{\sum_{k=1}^{n} \frac{2}{k} \log k}{(\log n)^{2}} \rightarrow 1
$$

the proof is complete.

Here we exhibited a strong law in the nonintegrable case. One can obtain similar strong laws for mean zero random variables when $P\{|X|>x\}=L(x) / x$ (see, e.g., Adler and Rosalsky [5]). 
Acknowledgement: The author wishes to thank the Referee for his/her valuable comments and helpful suggestions which led to an improved presentation of this paper.

\section{REFERENCES.}

1. Heyde, C.C., 1969, "A note concerning behaviour of iterated logarithm type," Proc. Amer. Math. Soc., 23:85-90.

2. Feller, W., An Introduction to Probability Theory and Its Applications, Vol. II, 2nd Ed., John Wiley, New York, 1971.

3. Adler, A., "Exact sequences for sums of independent random variables," in Almost Everywhere Convergence, Editors: G.A. Edgar and L. Sucheston, Academic Press, Boston (pp. 11-29), 1989.

4. Adler, A. and Rosalsky, A., 1987, "Some general strong laws for weighted sums of stochastically dominated random variables," Stochastic Anal. Appl. 5:1-16.

5. Adler, A. and Rosalsky, A., 1987, "On the strong law of large numbers for normed weighted sums of i.i.d. random variables," Stochastic Anal. Appl. 5:467-483. 


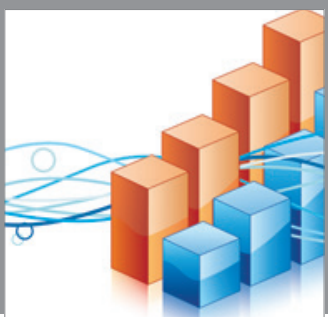

Advances in

Operations Research

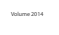

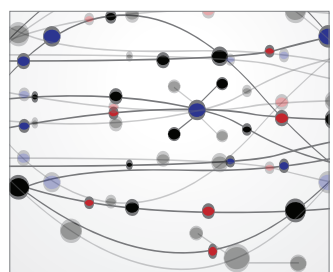

\section{The Scientific} World Journal
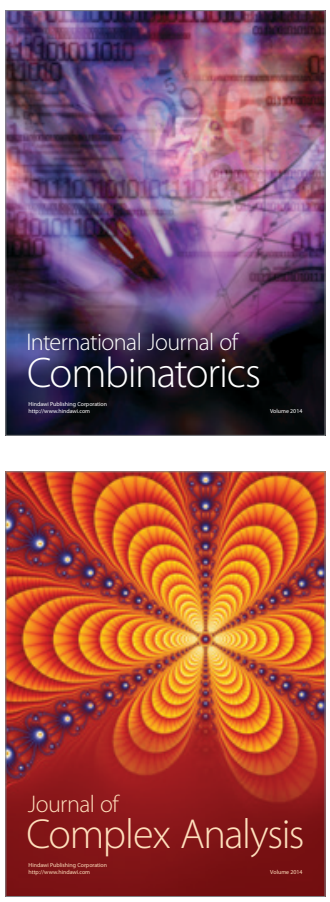

International Journal of

Mathematics and

Mathematical

Sciences
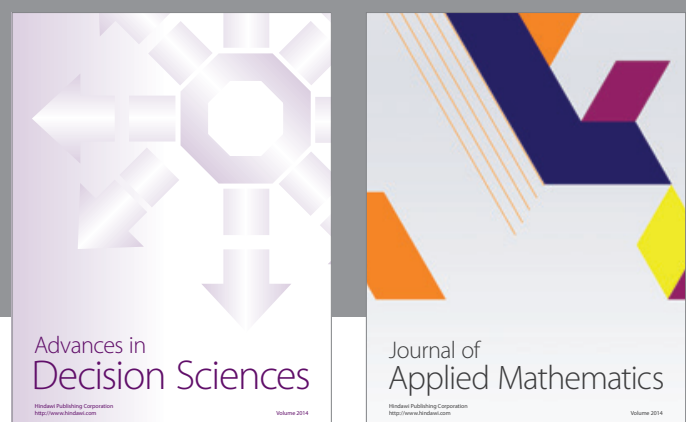

Journal of

Applied Mathematics
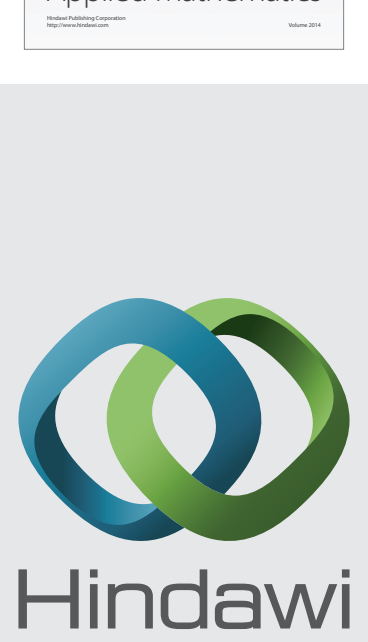

Submit your manuscripts at http://www.hindawi.com
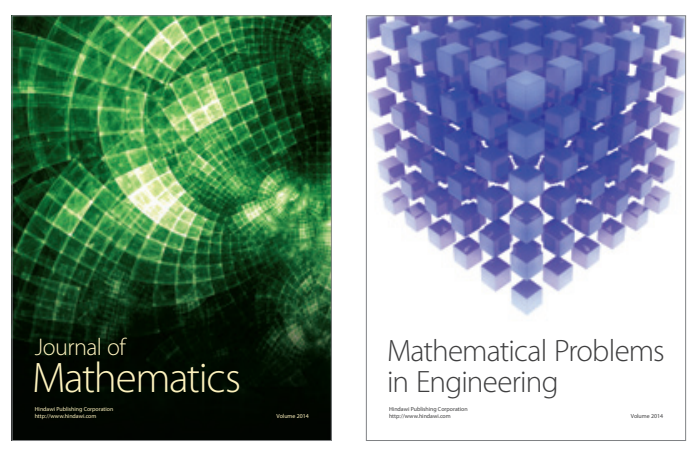

Mathematical Problems in Engineering
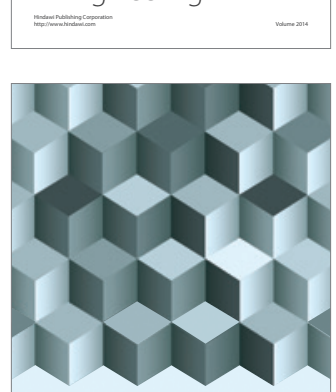

Journal of

Function Spaces
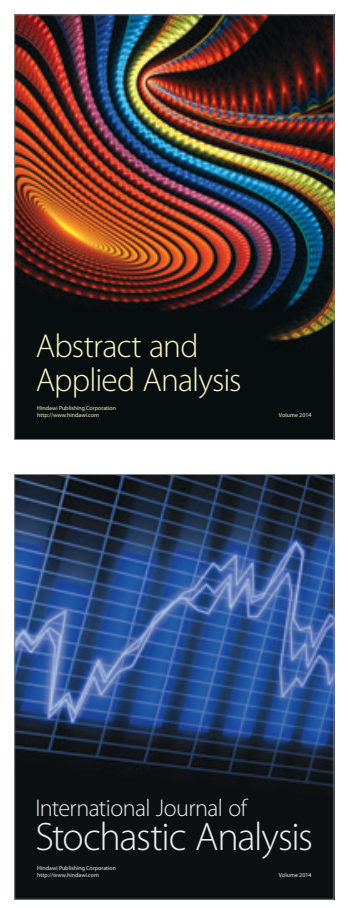

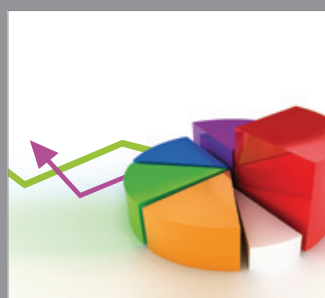

ournal of

Probability and Statistics

Promensencen
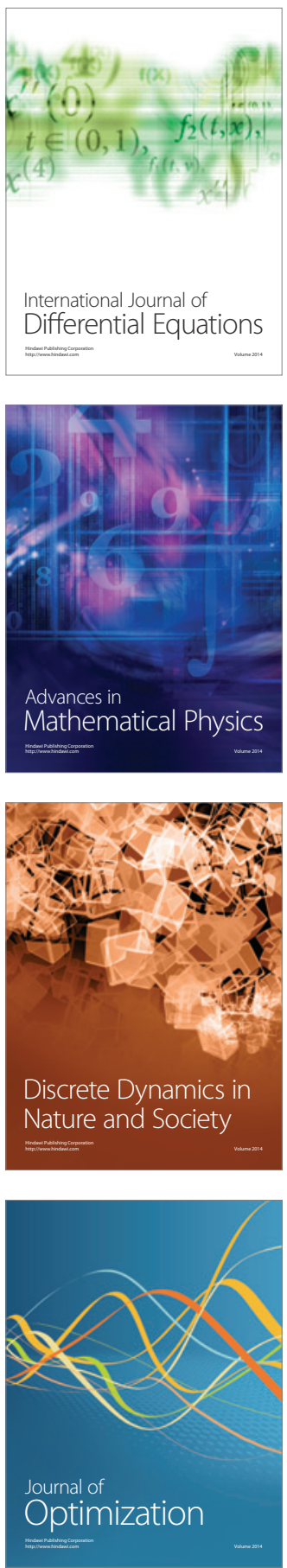